\title{
Optical Measurement of Blood Oxygen Saturation of Dental Pulp
}

\author{
Satoko Kakino, ${ }^{1}$ Shinya Kushibiki, ${ }^{2}$ Azusa Yamada, ${ }^{2}$ Zenzo Miwa, \\ Yuzo Takagi, ${ }^{1}$ and Yuji Matsuura ${ }^{2}$ \\ ${ }^{1}$ Division of Oral Health Sciences, Tokyo Medical and Dental University, 1-5-45 Yushima, Tokyo 113-0034, Japan \\ ${ }^{2}$ Graduate School of Biomedical Engineering, Tohoku University, 6-6-05 Aoba, Sendai 980-8579, Japan \\ Correspondence should be addressed to Satoko Kakino; satokopd.pedo@tmd.ac.jp
}

Received 13 August 2013; Accepted 24 September 2013

Academic Editors: D. Fotiadis and I. Toma-Dasu

Copyright (C) 2013 Satoko Kakino et al. This is an open access article distributed under the Creative Commons Attribution License, which permits unrestricted use, distribution, and reproduction in any medium, provided the original work is properly cited.

The applicability of arterial pulse oximetry to dental pulp was demonstrated using in vitro and in vivo measurements. First, porcine blood of known oxygen saturation $\left(\mathrm{SO}_{2}\right)$ was circulated through extracted human upper incisors, while transmitted-light plethysmography was performed using three different visible wavelengths. From the light intensity waveforms measured in vitro, a parameter $R$ that is statistically correlated to $\mathrm{SO}_{2}$ was calculated using the pulsatile/nonpulsatile component ratios of two wavelengths for different $\mathrm{SO}_{2}$. Then, $R$ values were measured in vivo for living incisors, and the corresponding $\mathrm{SO}_{2}$ values were calculated using the results of in vitro measurements. The estimated $\mathrm{SO}_{2}$ values of the upper central incisors measured in vivo were from 71.0 to $92.7 \%$. This study showed the potential to measure the oxygen saturation changes to identify the sign of pulpal inflammation.

\section{Introduction}

Diagnosing a dental pulp condition is important in clinical practice, but it is hard because dental pulp is covered by calcified tissue which makes it difficult to get objective parameters of pathological conditions. Conventional vitality tests of dental pulp, which are based on sensory nerve response to thermal and electrical stimulation, have limitations in terms of accurate diagnosis $[1,2]$. Since these tests require a subjective response from the patient, they can result in false positives, particularly in case of the pediatric patient. In addition, with immature permanent or traumatized teeth, false negative results can occur due to increased sensory nerve thresholds.

The dental pulp microcirculation system plays an essential role in maintaining tooth homeostasis, and pulp vitality depends on the function of the vascular system rather than that of the neural response. Several noninvasive and objective methods have been proposed for assessing the circulatory status of dental pulp, including laser Doppler flowmetry [3, 4], dual wavelength spectrophotometry [5], and optical plethysmography $[6,7]$. We previously developed a method called "transmitted-light plethysmography (TLP)" that optically detects circulatory changes in the blood flow and investigated the applicability of TLP to the vitality test of traumatized teeth [8]. It works by detecting the pulsation intensity of light transmitted through a tooth. It uses light with a wavelength of $525 \mathrm{~nm}$ from an LED and visualizes the pulpal blood flow synchronized with the heartbeat. We have used TLP in clinical practice to examine pulp vitality in traumatized teeth and found that the pulse amplitudes (pulse shape characteristics) change gradually over the course of clinical follow-ups. These findings suggest that local infections induced by trauma or caries might affect the acidity or metabolite of the pulp tissues, resulting in changes in the pulpal blood oxygen level.

Several groups have reported the application of a pulse oximeter, which is widely used to monitor arterial blood oxygen saturation $\left(\mathrm{SO}_{2}\right)$, to dental pulp. Schnettler and Wallace found that a pulse oximeter was effective in distinguishing vital and nonvital teeth [9], and Goho [10] and Radhakrishnan et al. [11] described how its objectivity is advantageous for pediatric patients. Kahan et al. [12] and Makiniemi et al. [13] measured $\mathrm{SO}_{2}$ by using a reflectance probe and found that it was difficult to detect tooth plethysmograms. Despite the recent report of oxygen saturation of around $80 \%$ as measured with a common oximeter with red and infrared 
LEDs [14], the absolute measurement of $\mathrm{SO}_{2}$ in dental pulp using standard pulse oximetry techniques is difficult [15]. This is because of the very small volume of arterial blood in dental pulp, which makes the amplitude ratios between the arterial pulsatile component and the nonpulsatile component much smaller than those measured for the fingertip or ear lobe. Therefore, a common oximeter designed for high blood volume is not well suited to tooth plethysmography.

In the present study, we experimentally measured amplitude ratios between the pulsatile and nonpulsatile components by using an extracted tooth model with a circulating flow of blood for which the $\mathrm{SO}_{2}$ value was precisely controlled. The blood samples used had a hematocrit of $3 \%$, which we estimated from a previous study of blood in the pulp chamber [16]. Three wavelengths $(450-600 \mathrm{~nm})$ in the visible region were used for detection as blood shows much higher absorption at such wavelengths [15-17]. The longer red and near-infrared wavelengths that are commonly used in pulse oximeters for fingertip measurement are not effective for detecting the slight amount of blood in the pulp because of the relatively small absorption of hemoglobin at these wavelengths. In addition, since the dentin and enamel surrounding the pulp have high transmission for blue to orange wavelengths, these shorter wavelengths are more appropriate for tooth plethysmography. Then absolute $\mathrm{SO}_{2}$ values for human dental pulp were estimated from in vivo measurement.

\section{Application of Pulse Oximetry to Dental Pulp}

2.1. Principle of $\mathrm{SO}_{2}$ Measurement. Pulse oximetry utilizes optical plethysmograms measured at two different wavelengths. Light is absorbed by the pulsating arterial blood, nonpulsating arterial blood, venous blood, and various tissues (dentin, enamel, and connective). The signals detected using light transmitted through a tooth consist of a bias nonpulsatile component with an intensity of $I_{B}$ and a pulsatile component (known as a plethysmogram) with a vibrational amplitude of $I_{P}$ that is superimposed on the bias component.

To calculate the oxygen saturation, we first calculated $I_{P} / I_{B}$ for wavelengths $\lambda_{1}$ and $\lambda_{2}$ to obtain parameter $R$ [18]:

$$
R=\frac{I_{P} /\left.I_{B}\right|_{\lambda=\lambda_{1}}}{I_{P} /\left.I_{B}\right|_{\lambda=\lambda_{2}}} .
$$

In general pulse oximetry, $R$ is a variable parameter of $\mathrm{SO}_{2}$ values. It is usually expressed by a polynomial equation as follows:

$$
\mathrm{SO}_{2}=f(R)
$$

The constants in the equation are related to the wavelengths, absorption coefficients of the oxy- and deoxyhemoglobin, and other factors [18]. Therefore, any $\mathrm{SO}_{2}$ level can be estimated by using (2). Since there is an assumption underlying (1) that scattering and absorption coefficients are constants between two wavelengths, we first measured the attenuation constants of enamel and dentin to show the validity of (1).

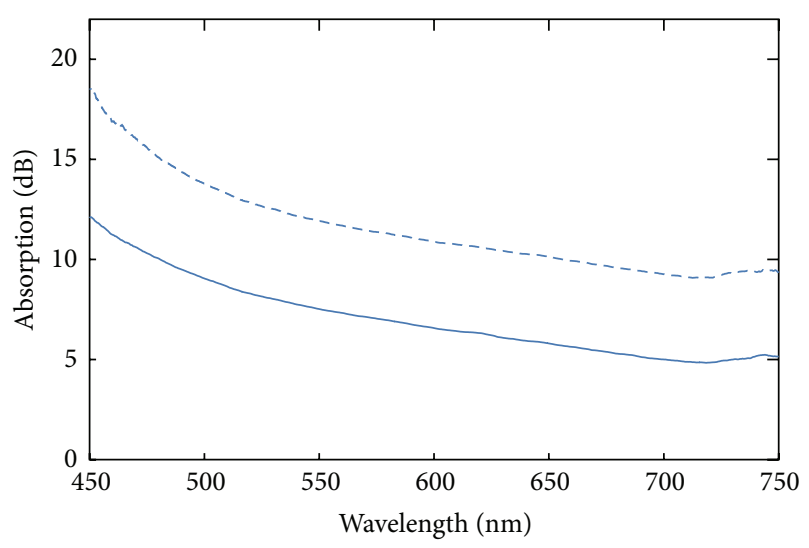

Tooth no. 1

— Tooth thickness $2 \mathrm{~mm}$

- - - Tooth thickness $3 \mathrm{~mm}$

FIGURE 1: Absorption spectra of sliced-tooth samples with different thicknesses.

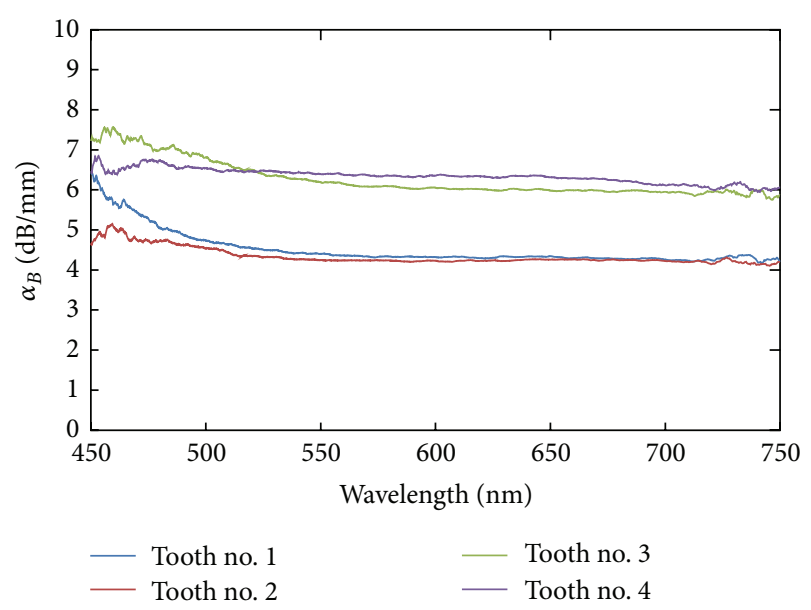

Figure 2: Attenuation spectrum of sliced-tooth samples.

2.2. Measurement of Attenuation Constants. Tooth section samples of two different thicknesses $(2.0$ and $3.0 \mathrm{~mm})$ were cut from four extracted human upper incisors including central and lateral incisors, in which dental trauma often occurs. The thickness of the enamel for each sample was $0.5 \mathrm{~mm}$, and the total thickness of the dentin was 2.0 and $3.0 \mathrm{~mm}$. This is because tooth size mainly depends on the dentin thickness, while the enamel thickness is almost constant. The samples were illuminated by a white light source through a bundled poly(methyl methacrylate) (PMMA) optical fiber with a total diameter of $1.6 \mathrm{~mm}$. Such fiber has high transmission efficiency and flexibility. The transmitted light was guided to the spectrophotometer (USB2000, Ocean Optics) by using another bundled fiber.

Absorption spectra of one of the samples are shown in Figure 1. In the both samples with different thicknesses, the attenuation was high in short wavelengths because of the large scattering effect, and the other tooth samples showed 


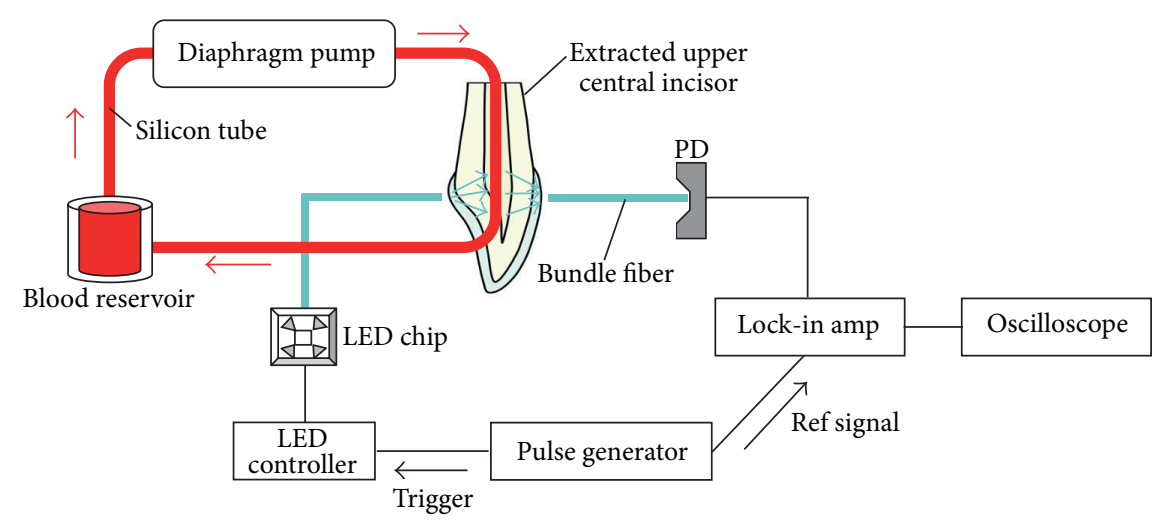

FIGURE 3: Optical measurement set-up for pulpal circulation model.

the same tendency. From these spectra, we calculated the attenuation coefficients using the cutback method. Using this method eliminated the effect of surface reflectance and scattering and thus the reflectance at the boundary between the enamel and dentin. Figure 2 shows the calculated attenuation constants $\alpha_{B}$ of the samples. Although the level of the attenuation differs in each tooth sample and slight increases were observed in short wavelengths, the attenuation coefficients were almost constant in the wavelength range of 450 to $750 \mathrm{~nm}$. From this result we confirmed that the assumption underlying (1) was supported.

\section{Optical Measurement Set-Up for Pulpal Circulation Model}

To find parameter $R$ for (1) and to specify the function $f$ in (2), which is needed to estimate pulpal $\mathrm{SO}_{2}$, we set up a pulpal circulation model, as shown in Figure 3. An extracted human upper incisor that was free of caries, restoration, and defects was prepared for use as an in vitro circulation model. The buccolingual thickness of the sample tooth was $6 \mathrm{~mm}$ which was the average size of the upper central incisors [16]. The tooth was cut approximately $3 \mathrm{~mm}$ above the root apex using a dental air turbine hand-piece with a diamond point, and the root canal was enlarged to $3 \mathrm{~mm}$ in diameter to enable a silicone tube $(3 \mathrm{~mm} / 2 \mathrm{~mm}$ outer/inner diameters $)$ to be inserted. A simulated pulsating blood flow with a pulse rate of $1 \mathrm{~Hz}$ was created in the silicon tube by using a diaphragm pump (Masterflex, Cole-Parmer).

An LED with a peak emission wavelength of 525 or $590 \mathrm{~nm}$ and a bandwidth of around $35 \mathrm{~nm}$ was used as the light source. The sample tooth was illuminated from the palatal side using the bundled optical fiber described above, and the light transmitted through the tooth was guided to a photodiode using another optical fiber, as described above. The output power from the illuminating bundled fiber was 0.2-0.3 mW, which did not affect the pulp viability. The two optical fibers were aligned perpendicular to the longitudinal axis of the tooth. To eliminate background noise, the incident light was modulated at $1 \mathrm{kHz}$ with a duty cycle of $10 \%$ to reduce the total power to suppress the heat generation, and the transmitted light was detected with a lock-in amplifier synchronized with the light source pulses.

Fresh porcine blood that had been anticoagulated with acid citrate dextrose (ACD-A solution, Terumo) was centrifuged and washed with phosphate buffered saline solution ( $\mathrm{PBS}, \mathrm{pH}=7.4$ ) to remove the blood plasma. The hematocrit of the blood sample was adjusted to $3 \%$ with PBS by referring to the reported effective hematocrit of pulpal blood [16]. The blood was oxygenated by bubbling it with a gas mixture $(96 \%$ $\mathrm{O}_{2} / 4 \% \mathrm{CO}_{2}$ ) and deoxygenated with another gas mixture $\left(96 \% \mathrm{~N}_{2} / 4 \% \mathrm{CO}_{2}\right)$. The blood $\mathrm{SO}_{2}$ level, which ranged from 18 to $95 \%$, was measured with a blood gas analyzer (GASTATmini, Techno Medica).

\section{Results and Discussion}

Here, we firstly discuss the validity of the in vitro model for the estimation of $\mathrm{SO}_{2}$ values and then, using the $R$ values measured by the model, show some in vivo results of measurement of blood oxygen saturation of dental pulp. One of the advantages of the in vitro model is that light attenuation in the teeth is not influenced by the surrounded periodontal structures or oral soft tissues. Therefore, in contrast to in vivo measurements where a variety of disturbances should be taken into account, one can precisely measure a wide range of blood $\mathrm{SO}_{2}$ values.

However, there are some limitations in this model. In human teeth, it is supposed that arterioles in dental pulp mainly contribute to pulsatile changes of light transmitted through the teeth. In the in vitro model, the pulpal microcirculation system consisting of arterioles and venous and lymph vessels cannot be replicated. However, we speculate that the inhomogeneous structures inside the pulp chamber were simplified by whole blood since inflammatory conditions such as pulpitis or partial pulp necrosis easily spread to whole pulp and alter the blood $\mathrm{SO}_{2}$ because of the narrow pulp chamber surrounded by hard tissues.

The pulsatile component $I_{P}$ and nonpulsatile component $I_{B}$ in optical plethysmogram at the 470-, 525-, and 590- nm wavelengths were recorded with an oscilloscope for different blood $\mathrm{SO}_{2}$ values. These measurement wavelengths were chosen on the basis of three conditions: (i) sensitivity to the 


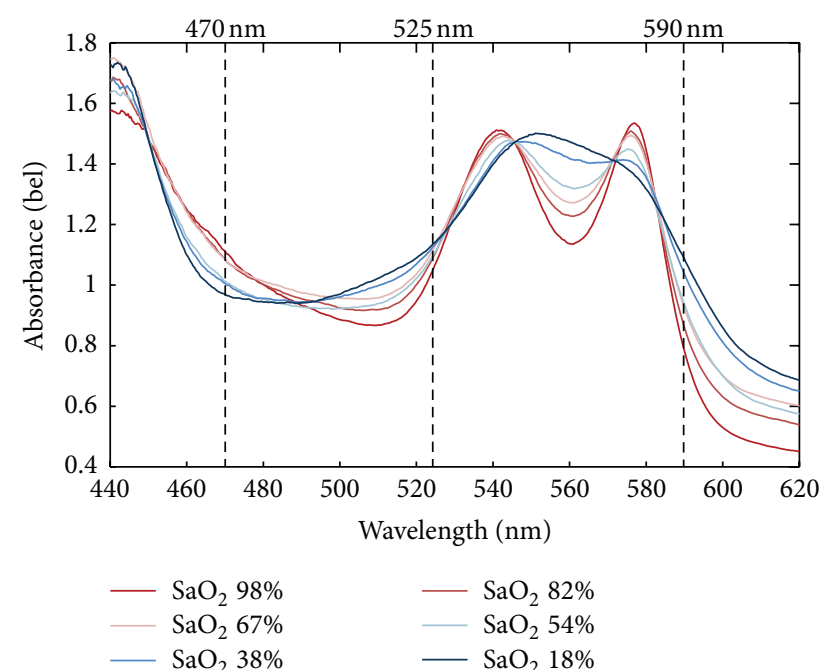

FIgURE 4: Absorption spectra of hemoglobin with different $\mathrm{SO}_{2}$ levels.

volumetric change of pulpal blood flow, (ii) sensitivity to changes in pulpal blood oxygen saturation, and (iii) transmissivity in hard tissue. In terms of sensitivity to the blood volume variation, both in vivo and in vitro studies have shown that higher pulsatile/nonpulsatile ratios can be detected with a green LED than with red and infrared LEDs [15-17].

Figure 4 shows absorption spectra of hemoglobin solution with different blood $\mathrm{SO}_{2}$ levels. From this figure, one can easily find that light with wavelengths shorter than $600 \mathrm{~nm}$ has high sensitivity to the volumetric change of pulpal blood flow because of the high absorption coefficients. We also confirmed that these shorter wavelengths are appropriate to the measurement of the tooth plethysmograms from human incisors. Concerning the transmissivity in hard tissue, enamel and dentin are transparent enough for the detection of tooth plethysmograms at wavelengths between 450 and $600 \mathrm{~nm}$ as shown in Figure 2.

With respect to the sensitivity to blood $\mathrm{SO}_{2}$ level changes, Figure 4 indicates that nonisosbestic wavelengths of $470 \mathrm{~nm}$ and $590 \mathrm{~nm}$ are highly sensitive to changes in $\mathrm{SO}_{2}$ levels. When the $\mathrm{SO}_{2}$ level increases, absorbance at $470 \mathrm{~nm}$ also increases and absorbance at $590 \mathrm{~nm}$ shows the opposite trend. Therefore, by combining these wavelengths with an isosbestic wavelength of $525 \mathrm{~nm}$, one can perform a precise measurement of $\mathrm{SO}_{2}$ levels.

As shown in Figure 5 , the $I_{P} / I_{B}$ ratios linearly decreased for $470 \mathrm{~nm}$ and $525 \mathrm{~nm}$ and increased for $590 \mathrm{~nm}$ as $\mathrm{SO}_{2}$ increased, as theoretically predicted. The correlation coefficients $r$ for the slope were higher than absolute value of 0.70 despite the presence of dentin and enamel. They were -0.89 , -0.70 , and 0.86 , respectively. The results in Figure 5 show that all three wavelengths have sufficient sensitivity for detecting plethysmograms. The green wavelength, $525 \mathrm{~nm}$, coincides with one of the isosbestic wavelengths of hemoglobin and showed the lowest correlation coefficient. The orange and blue wavelengths, 590 and $470 \mathrm{~nm}$, are more suitable for $\mathrm{SO}_{2}$

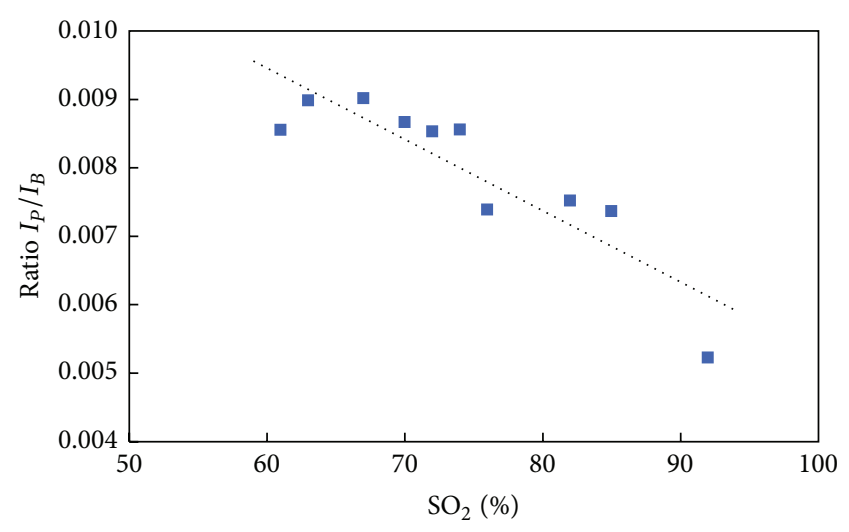

(a)

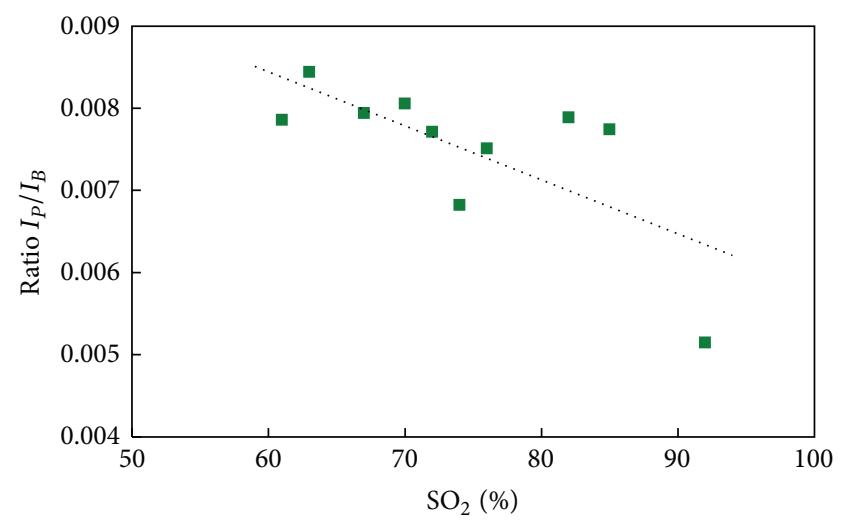

(b)

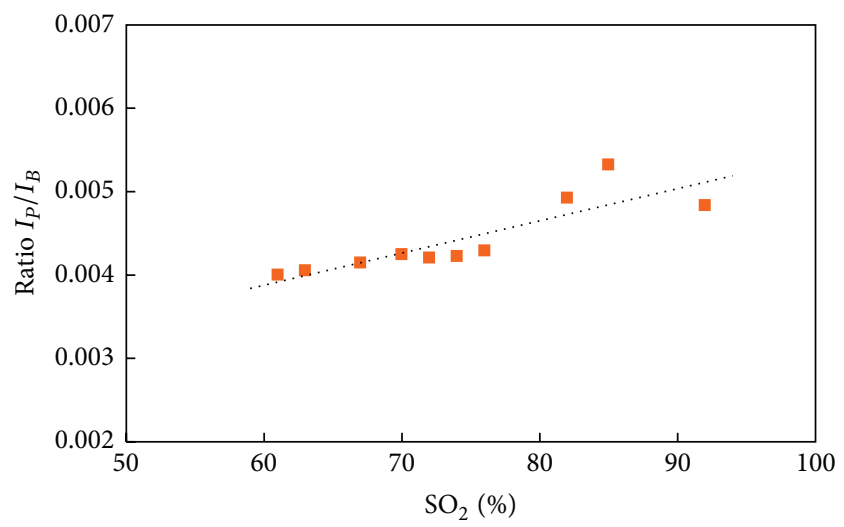

(c)

Figure 5: Measured ratios between pulsatile component $I_{P}$ and nonpulsatile component $I_{B}$ as a function of $\mathrm{SO}_{2}$ values for (a) 470, (b) 525 , and (c) $590 \mathrm{~nm}$.

measurement of dental pulp because of its high sensitivity to changes in the $\mathrm{SO}_{2}$ level.

The $R$ values in (1) calculated for the 525/590 and $470 / 590 \mathrm{~nm}$ combinations are shown in Figure 6 with second-order regression lines. The $R$ values were statistically correlated with the $\mathrm{SO}_{2}$ values $(P<0.01)$, and the correlation for regression curve was 0.88 and 0.99 , respectively. The higher correlation of $470 / 590 \mathrm{~nm}$ combinations means that both of the nonisosbestic wavelengths have high sensitivity to the $\mathrm{SO}_{2}$ changes in the dental pulp. 


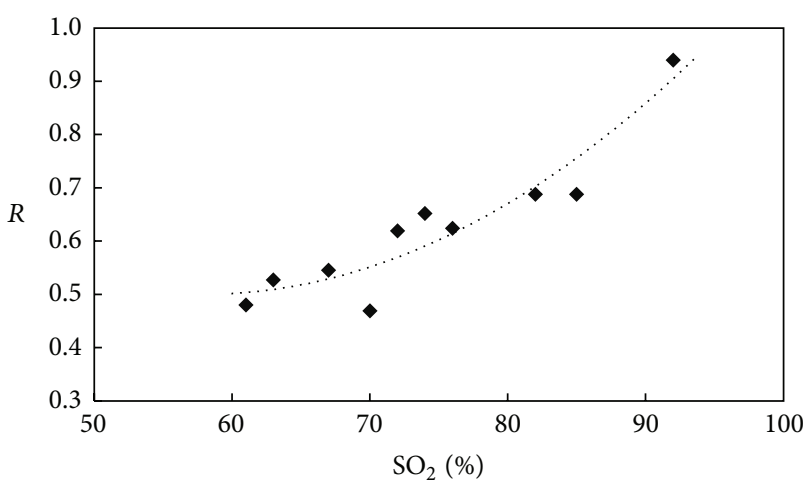

(a)

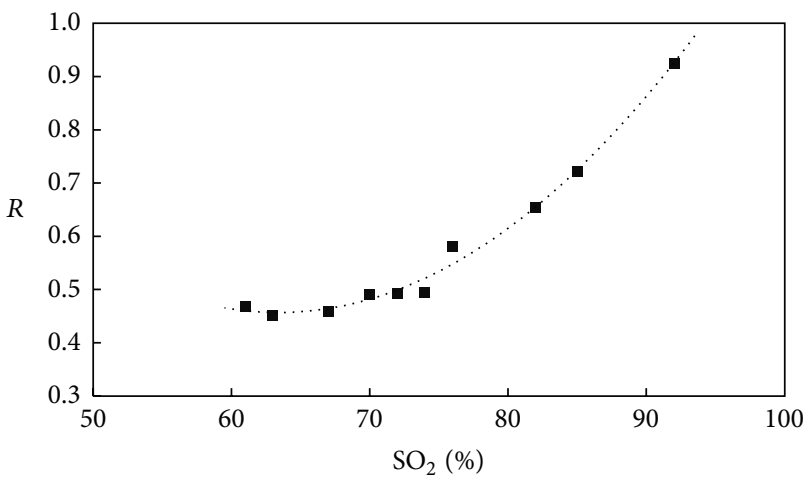

(b)

FIGURE 6: $R$ values versus $\mathrm{SO}_{2}$ calculated from results for (a) 525/590 and (b) $470 / 590 \mathrm{~nm}$ combinations.

TABLE 1: Summary of the in vivo $\mathrm{SO}_{2}$ estimation.

\begin{tabular}{lccc}
\hline Subject & Age & $R(470 / 590 \mathrm{~nm})$ & $\mathrm{SO}_{2}$ estimate $(\%)$ \\
\hline $\mathrm{A}$ & 26 & 0.75 & 85.8 \\
$\mathrm{~B}$ & 27 & 0.95 & 92.7 \\
$\mathrm{C}$ & 28 & 0.79 & 87.3 \\
$\mathrm{D}$ & 28 & 0.80 & 87.9 \\
$\mathrm{E}$ & 30 & 0.49 & 71.0 \\
$\mathrm{~F}$ & 32 & 0.63 & 80.9 \\
$\mathrm{G}$ & 32 & 0.87 & 90.1 \\
\hline Mean & & 0.75 & 85.1 \\
S.D. & & 0.15 & 7.2
\end{tabular}

For the reference to validate the in vitro study, we examined healthy upper central incisors of volunteers aged from 26 to 32 years $(n=7)$. After optically measuring $I_{P}$ and $I_{B}$ at 470 and $590 \mathrm{~nm}$, we calculated the $R$ value using (1) and found that it was from 0.49 to 0.95 . Using the regression curve of $R$ versus $\mathrm{SO}_{2}$ derived from the results shown in Figure 6(b), we calculated the estimated $\mathrm{SO}_{2}$ value for the $470 / 590 \mathrm{~nm}$ combination and found that it was 71.0 to $92.7 \%$ as shown in Table 1 . We speculated that pulpal $\mathrm{SO}_{2}$ values of the healthy teeth were lower than systemic arterial $\mathrm{SO}_{2}$ values, because it might be more affected by the venule oxygen level due to the pulpal narrow noncompliance structure. The results of our study were supported by this discussion.

\section{Conclusion}

We have demonstrated the applicability of pulse oximetry to dental pulp by using an in vitro blood circulation model. The light source was a power LED with a peak emission wavelength of 470,525 , or $590 \mathrm{~nm}$. There was a linear correlation between the pulsatile/nonpulsatile component ratios and the pulpal oxygen saturation $\left(\mathrm{SO}_{2}\right)$ values for the three wavelengths. The nonisosbestic wavelengths, 470 and $590 \mathrm{~nm}$ (blue and orange), showed higher sensitivity than the isosbestic wavelength of $525 \mathrm{~nm}$ to changes in pulpal $\mathrm{SO}_{2}$. The $R$ parameter values calculated from the pulsatile/nonpulsatile component ratios of two different wavelengths were statistically correlated to pulpal $\mathrm{SO}_{2}$. In addition, the measured attenuation coefficients of enamel and dentin were almost constant in the visible wavelength range, which supports the validity of using pulse oximetry for dental pulp. The estimated $\mathrm{SO}_{2}$ values measured in vivo were 71.0 to $92.7 \%$. Diagnosis of pulpal condition in relation to pulpal inflammation due to caries or dental trauma may be feasible by using the pulse oximetry to dental pulp. We are working on the investigation of the effects of various pathological conditions on pulpal $\mathrm{SO}_{2}$, and the results will be reported elsewhere.

\section{Conflict of Interests}

The authors declare that there is no conflict of interests regarding the publication of this paper.

\section{Acknowledgment}

This study was partially supported by a Grant-in-Aid for Young Scientists (B) no. 21791843 from the Ministry of Education, Culture, Sports, Science and Technology of Japan.

\section{References}

[1] H. J. Fulling and J. O. Andreasen, "Influence of maturation status and tooth type of permanent teeth upon electrometric and thermal pulp testing," Scandinavian Journal of Dental Research, vol. 84, no. 5, pp. 286-290, 1976.

[2] H. Klein, "Pulp responses to an electric pulp stimulator in the developing permanent anterior dentition," ASDC Journal of Dentistry for Children, vol. 45, no. 3, pp. 199-202, 1978.

[3] B. Gazelius, L. Olgart, B. Edwall, and L. Edwall, "Non-invasive recording of blood flow in human dental pulp," Endodontics \& Dental Traumatology, vol. 2, no. 5, pp. 219-221, 1986.

[4] B. Gazelius, L. Olgart, and B. Edwall, "Restored vitality in luxated teeth assessed by laser Doppler flowmeter," Endodontics \& Dental Traumatology, vol. 4, no. 6, pp. 265-268, 1988.

[5] R. Nissan, M. Trope, C. D. Zhang, and B. Chance, "Dual wavelength spectrophotometry as a diagnostic test of the pulp chamber contents," Oral Surgery Oral Medicine and Oral Pathology, vol. 74, no. 4, pp. 508-514, 1992.

[6] I. Shoher, Y. Mahler, and S. Samueloff, "Dental pulp photoplethysmography in human beings," Oral Surgery Oral Medicine and Oral Pathology, vol. 36, no. 6, pp. 915-921, 1973.

[7] M. Ikawa, H. Horiuchi, and K. Ikawa, "Optical characteristics of human extracted teeth and the possible application of 
photoplethysmography to the human pulp," Archives of Oral Biology, vol. 39, no. 10, pp. 821-827, 1994.

[8] Z. Miwa, M. Ikawa, H. Iijima, M. Saito, and Y. Takagi, "Pulpal blood flow in vital and nonvital young permanent teeth measured by transmitted-light photoplethysmography: a pilot study," Pediatric Dentistry, vol. 24, no. 6, pp. 594-598, 2002.

[9] J. M. Schnettler and J. A. Wallace, "Pulse oximetry as a diagnostic tool of pulpal vitality," Journal of Endodontics, vol. 17, no. 10, pp. 488-490, 1991.

[10] C. Goho, "Pulse oximetry evaluation of vitality in primary and immature permanent teeth," Pediatric Dentistry, vol. 21, no. 2, pp. 125-127, 1999.

[11] S. Radhakrishnan, A. K. Munshi, and A. M. Hegde, "Pulse oximetry: a diagnostic instrument in pulpal vitality testing," The Journal of Clinical Pediatric Dentistry, vol. 26, no. 2, pp. 141-145, 2002.

[12] R. S. Kahan, K. Gulabivala, M. Snook, and D. J. Setchell, "Evaluation of a pulse oximeter and customized probe for pulp vitality testing," Journal of Endodontics, vol. 22, no. 3, pp. 105109, 1996.

[13] M. Makiniemi, H. K. Kopola, K. Oikarinen, and E. Herrala, "Novel fiber optic dental pulp vitalometer," in Medical Sensors II and Fiber Optic Sensors, Proceedings of SPIE, pp. 140-148, September 1994.

[14] M. H. Pozzobon, R. de Sousa Vieira, A. M. H. Alves et al., "Assessment of pulp blood flow in primary and permanent teeth using pulse oximetry," Dental Traumatology, vol. 27, no. 3, pp. 184-188, 2011.

[15] J. M. Schmitt, R. L. Webber, and E. C. Walker, "Optical determination of dental pulp vitality," IEEE Transactions on Biomedical Engineering, vol. 38, no. 4, pp. 346-352, 1991.

[16] S. Kakino, Y. Takagi, and S. Takatani, "Absolute transmitted light plethysmography for assessment of dental pulp vitality through quantification of pulp chamber hematocrit by a threelayer model," Journal of Biomedical Optics, vol. 13, no. 5, Article ID 054023, 2008.

[17] M. E. Fein, A. H. Gluskin, W. W. Y. Goon, B. B. Chew, W. A. Crone, and H. W. Jones, "Evaluation of optical methods of detecting dental pulp vitality," Journal of Biomedical Optics, vol. 2, no. 1, pp. 58-73, 1997.

[18] I. Yoshiya, Y. Shimada, and K. Tanaka, "Spectrophotometric monitoring of arterial oxygen saturation in the fingertip," Medical and Biological Engineering and Computing, vol. 18, no. 1, pp. 27-32, 1980. 

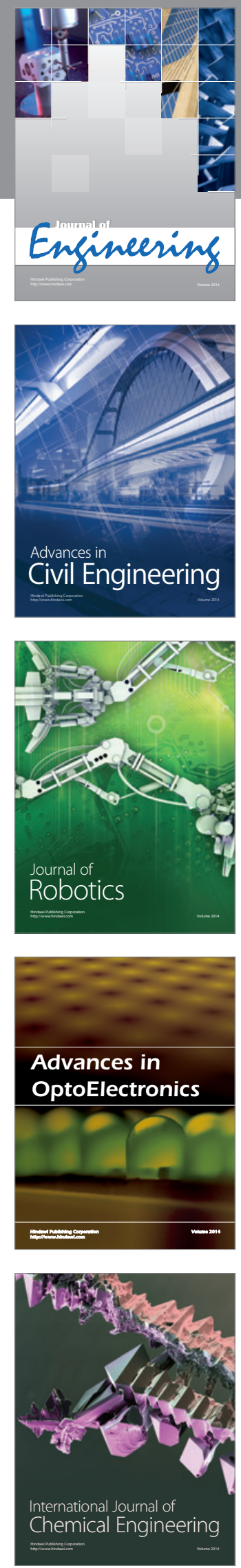

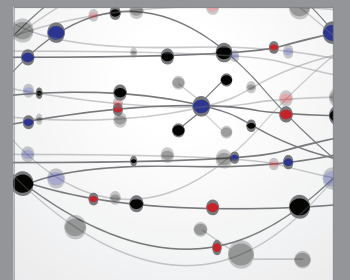

The Scientific World Journal
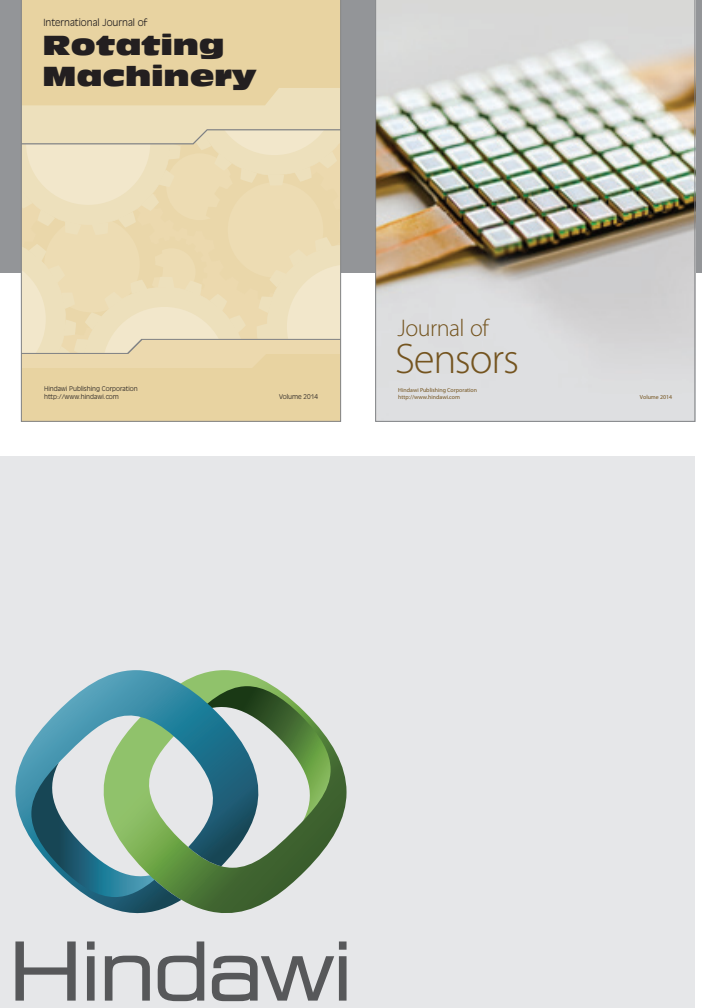

Submit your manuscripts at http://www.hindawi.com
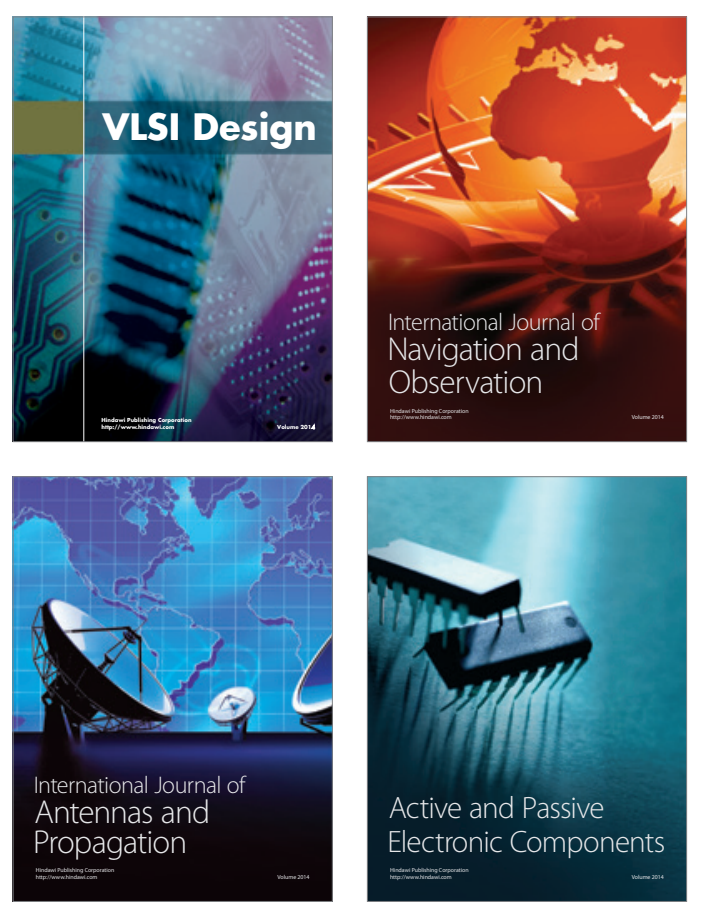
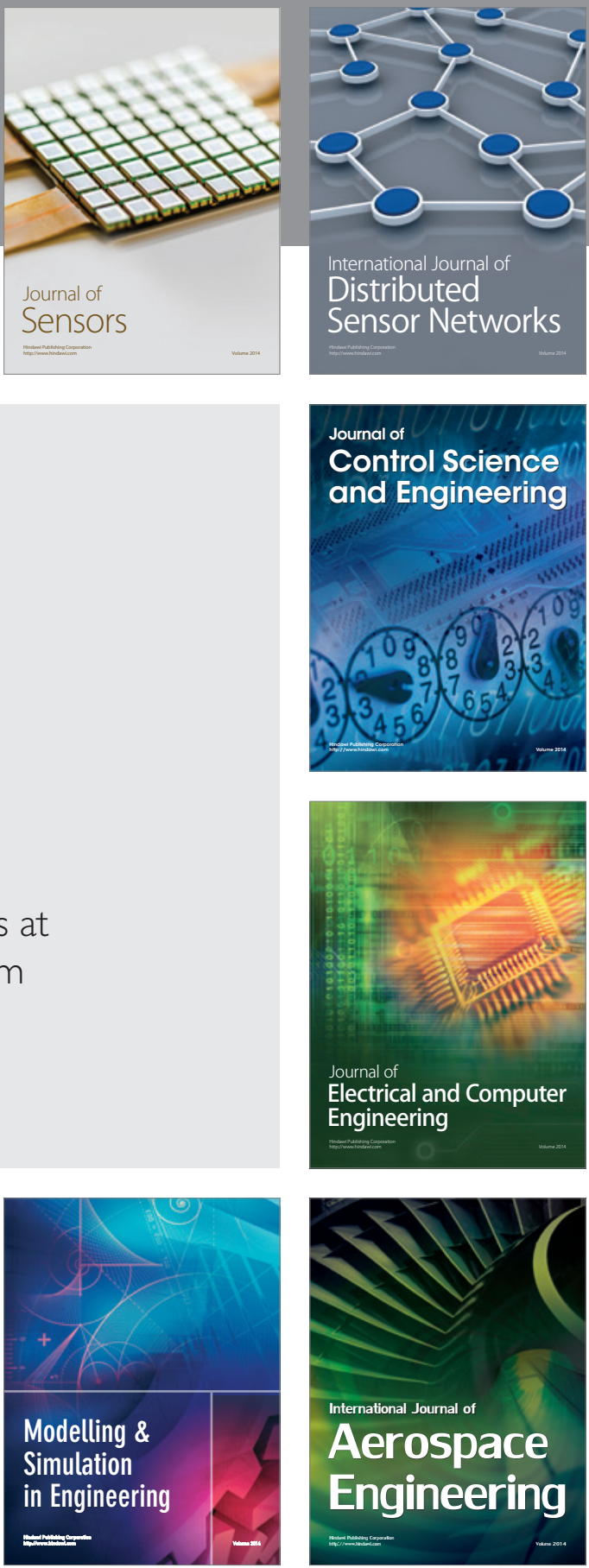

Journal of

Control Science

and Engineering
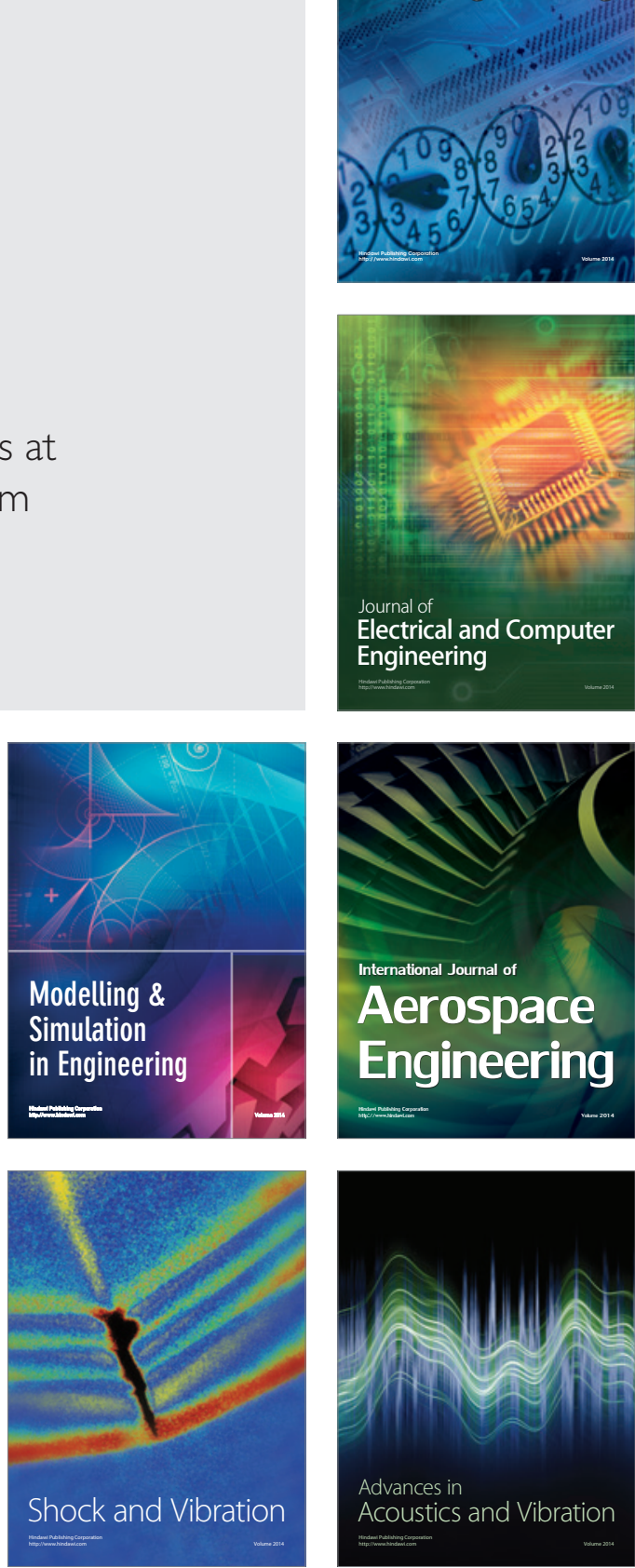